\title{
Processamento sensorial e desenvolvimento cognitivo de lactentes nascidos pré-termo e a termo
}

\author{
Flávia Regina Ribeiro Cavalcanti Buffone ${ }^{a}$, Sophie Helena Eickman ${ }^{b}$, \\ Marília de Carvalho Limab
}

aDepartamento de Terapia Ocupacional, Universidade Federal da Paraíba - UFPB, João Pessoa, PB, Brasil.

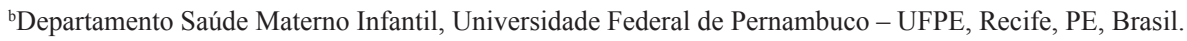

\begin{abstract}
Resumo: Introdução: Estudos atuais apontam para as repercussões que as alterações de processamento sensorial trazem para o desenvolvimento neuropsicomotor infantil. Pouco se conhece sobre a influência dessas alterações no desenvolvimento cognitivo de lactentes, porém sabe-se que estas interferem negativamente no desempenho das atividades cotidianas e trazem prejuízos ao longo da vida do indivíduo. Objetivo: Avaliar a relação entre o processamento sensorial e o desenvolvimento cognitivo de lactentes, e a associação entre a prematuridade e o processamento sensorial dessa população. Método: Estudo de corte transversal realizado no Ambulatório de Puericultura do Hospital das Clínicas da UFPE, no período de dezembro de 2009 a agosto de 2010. A amostra consistiu de 182 lactentes de oito a 15 meses de idade, dos quais 54 (29,7\%) nasceram prematuros, sendo feita a correção da prematuridade para 40 semanas de idade gestacional. Utilizou-se o Test of Sensory Functions in Infants (TSFI), para avaliar o processamento sensorial, e a Bayley Scales of Infant and Toddler Development III, na avaliação do desenvolvimento cognitivo. Resultados: Verificou-se uma frequência significantemente maior de processamento sensorial em risco e deficiente entre os lactentes nascidos pré-termo (37\%) quando comparado ao processo sensorial dos nascidos a termo $(21,9 \%)$. $\mathrm{O}$ atraso cognitivo foi significantemente maior $(8,3 \%)$ entre os lactentes com processamento sensorial em risco e deficiente em relação aos com processamento sensorial normal $(1,5 \%)$. Conclusão: A prematuridade foi considerada um fator de risco para distúrbio do processamento sensorial e os lactentes diagnosticados com este distúrbio apresentaram atraso cognitivo mais frequentemente. A prematuridade, isoladamente, não esteve associada ao atraso cognitivo.
\end{abstract}

Palavras-chave: Desenvolvimento Infantil, Processamento Sensorial, Neonatologia, Pediatria, Prematuro, Terapia Ocupacional.

\section{Sensory processing and cognitive development of preterm and full term infants}

\begin{abstract}
Introduction: Current studies show the repercussion of sensory processing disorder in infant neurodevelopment. Little is known about the influence of these disorders in the infant's cognitive development, however, it is known that they negatively interfere on daily life activities and remain during life course. Objective: To evaluate the relationship between sensory processing and cognitive development in infants and the association between prematurity and sensory processing in this population. Method: This is a cross-sectional study conducted in the Childcare Outpatient Department of the Hospital das Clínicas, Federal Universidade de Pernambuco, from December 2009 to August 2010. The sample consisted of 182 infants from 8 to 15 months, of which 54 (29.7\%) were born preterm with the prematurity age correction made to 40 weeks of gestational age. We used the Test of Sensory Functions in Infants (TSFI) to evaluate the sensory processing and the Bayley Scales of Infant and Toddler Development III to assess cognitive development. Results: There was a significantly higher frequency of at risk
\end{abstract}

Autor para correspondência: Flávia Regina Ribeiro Cavalcanti Buffone, Departamento de Terapia Ocupacional, Centro de Ciências da Saúde, Universidade Federal da Paraíba, Cidade Universitária, s/n, Castelo Branco, CEP 58051-900, João Pessoa, PB, Brasil, e-mail: flaviacavalcanti_to@ yahoo.com.br; flaviarre@hotmail.com

Recebido em: Set. 8, 2015; $1^{\text {a }}$ Revisão em Jan. 15, 2016; 2 ${ }^{\text {a }}$ Revisão em Abr. 6, 2016; $3^{\text {a }}$ Revisão em Jun. 18, 2016; $4^{\text {a }}$ Revisão em Ago. 4, 2016; Aceito em Ago. 22, 2016. 
and deficient sensory processing among preterm infants (37\%) when compared to term infants (21.9\%). Cognitive delay was significantly higher $(8.3 \%)$ in infants with at risk and deficient sensory processing when compared to those with normal sensory processing $(1.5 \%)$. Conclusion: Prematurity was a risk factor for sensory processing disorder, and infants diagnosed with this disorder showed cognitive delay more frequently. Prematurity alone was not associated with cognitive delay.

Keywords: Infant Development, Sensory Processing, Neonatology, Pediatrics, Preterm, Occupational Therapy.

\section{Introdução}

Estudos apontam para as repercussões que as alteraçôes de processamento sensorial trazem para o desenvolvimento neuropsicomotor infantil (BART et al., 2011; KOENIG; RUDNEY, 2010; MITCHELL et al., 2015; WICKREMASINGHE et al., 2013). Existe uma preocupação com a detecção precoce desses problemas, porém pouco se conhece sobre a influência dessas alteraçôes no desempenho cognitivo desses lactentes. Sabe-se que, nos primeiros dois anos de vida, o lactente aprende a detectar e interpretar as informaçóes sensoriais do tato, da posição e do movimento do corpo, da audição, da visão, do cheiro e do paladar. Essas informações são utilizadas para a aquisição de habilidades, como a manutenção da postura e do equilíbrio do corpo, a coordenação motora grossa e fina, e o desenvolvimento do esquema corporal (BRASIL, 2011; PEDROSA; CAÇOLA; CARVALHAL, 2015).

O desenvolvimento neuropsicomotor é influenciado pela associação de fatores biológicos com a qualidade da estimulação ambiental. Entre os fatores biológicos, está a prematuridade, considerada como condiçáo de risco para as alteraçóes no desenvolvimento de ordem cognitiva, motora, comportamental e de processamento sensorial, que podem ser ocasionados pela imaturidade da estrutura neurológica e influenciados pelas experiências sensoriais agressivas presentes no ambiente, mais especificamente, na unidade de terapia intensiva (UTI) neonatal (BART et al., 2011; WICKREMASINGHE et al., 2013; MITCHELL et al., 2015).

Os sintomas iniciais de alteração no processamento sensorial observados nos bebês foram estudados por Degangi (2000), que verificou uma possível relação entre estes e os problemas regulatórios, caracterizados por uma alta irritabilidade, dificuldade em se autoconsolar, choro excessivo e dificuldades com o sono e alimentação. Posteriormente, as crianças com problemas de processamento sensorial podem ter dificuldade em organizar açôes intencionais nas áreas da comunicação, da motricidade grossa e fina, e do brincar, apresentando também limitaçáo na participação social. Dificuldades com a leitura e a escrita também são frequentes nesses indivíduos, em idades mais avançadas (WIENER et al., 1996; BART et al., 2011).

Além dessas dificuldades, Koenig e Rudney (2010), em artigo de revisão sistemática, mostram que, em longo prazo, o desempenho nas atividades de vida diária e de vida prática, na participação social, no brincar e nas atividades acadêmicas também pode ser comprometido, dados corroborados por Mitchell et al. (2015). Os estudos realizados por Engel-Yeger (2008) e Bundy et al. (2007) mostram que as alteraçóes de modulação sensorial parecem ter mais influência nas escolhas por atividades do brincar e de lazer de crianças na idade escolar. As dificuldades encontradas por crianças dessa mesma faixa etária na execução das atividades de vida diária, como banho e alimentação, também foram associadas a problemas de processamento sensorial (WHITE et al., 2007). Mais tarde, o achado de Wickremasinghe et al. (2013) confirma que há uma tendência de as dificuldades no processamento sensorial ficarem mais evidentes, com o avanço da idade nas crianças. Já entre os adultos, Blanche (2005) verificou que as características do processamento sensorial permanecem ao longo da vida e que estas irão interferir nas escolhas das atividades ocupacionais e de lazer.

Algumas habilidades perceptocognitivas relacionadas ao sistema somatossensorial, como a identificação dos dedos e a grafestesia, foram medidas na idade escolar em crianças nascidas com extremo baixo peso, através do Sensory Integration and Praxis Tests (AYRES, 1989; DEMAIO-FELDMAN, 1994). Essas crianças apresentaram piores resultados nesses testes, quando comparadas com a população de peso adequado ao nascer, o que poderia ser justificado pelas experiências sensoriais dolorosas e pela restrição de posicionamento e movimento vivenciadas na UTI neonatal.

É no Ensino Fundamental que as dificuldades com a escola tendem a ficar mais evidentes. Segundo Reeves e Cermak (2002), os problemas que surgem estáo mais associados à práxis e, muitas vezes, o desempenho em tarefas, como escrita, recortar e colorir, e nos projetos de arte, é inferior ao das crianças sem alteração do processamento sensorial. 
Para os pais, esses escolares são caracterizados como distraídos ou descuidados, reforçando ainda mais as dificuldades apresentadas.

Case-Smith, Butcher e Reed (1998) verificaram o efeito da prematuridade no temperamento e no desenvolvimento de lactentes aos 12 meses de idade corrigida para os pré-termos e cronológica para os a termo, e sua associação com o processamento sensorial. Foi utilizada a escala Bayley II para medir o desenvolvimento mental e psicomotor, e a Sensory Rating Scale (SRS), que mede a resposta ao toque e ao movimento, ao som, ao estímulo visual e ao temperamento. Os lactentes nascidos prematuros apresentaram mais defensividade tátil e hiper-reatividade associada ao temperamento, quando comparados aos lactentes nascidos a termo; porém, nenhuma diferença foi observada no desenvolvimento mental ou psicomotor desses bebês.

Alguns autores procuraram conhecer as características do processamento sensorial ainda nos primeiros anos de vida do bebê, de maneira que alteraçôes do processamento sensorial possam ser detectadas precocemente e intervençóes possam ser iniciadas em momento oportuno, tentando prevenir possíveis complicaçôes para a vida futura da criança (MITCHELL, 2015; WICKREMASINGHE et al., 2013).

Ainda existe carência de pesquisas nesta área, indicando a necessidade da realização de mais estudos que avaliem possíveis associaçóes do processamento sensorial com outros componentes de desenvolvimento neuropsicomotor, a exemplo, a cogniçáo. Diante disso, o presente estudo teve por objetivo avaliar a associação entre a prematuridade e o processamento sensorial de lactentes, e a relaçáo entre o processamento sensorial e o desenvolvimento cognitivo dessa população.

\section{Método}

\subsection{Local e amostra do estudo}

A amostra foi composta por todos os lactentes nascidos prematuros (LNPT) e que frequentavam o Ambulatório do Recém-Nascido de Risco e de Puericultura do Hospital das Clínicas da Universidade Federal de Pernambuco (HC-UFPE), no período de dezembro de 2009 a agosto de 2010. Para cada LNPT recrutado, uma média de dois lactentes nascidos a termo (LNT), que eram acompanhados no Ambulatório de Puericultura do mesmo hospital, foram selecionados para formar o grupo controle. Dos 182 lactentes incluídos no estudo, 54 (30\%) eram lactentes nascidos pré-termo e 128 (70\%), lactentes nascidos a termo. No momento da avaliaçáo, todos estavam com idade entre 8 e 15 meses, sendo que, para os prematuros, foi utilizada a idade cronológica corrigida para 40 semanas. Os lactentes portadores de paralisia cerebral, perda auditiva, visão subnormal, deformidades, infecçóes congênitas, más formaçōes múltiplas ou síndromes genéticas foram excluídos do estudo.

O estudo foi aprovado pelo comitê de ética do Centro de Ciências da Saúde da UFPE (CAAE- 0217.0.000.172-09) e as mães ou responsáveis pelos lactentes, que se encontraram dentro dos critérios de inclusão, foram convidados a participar do estudo e assinaram o Termo de Consentimento Livre e Esclarecido.

\subsection{Instrumentos de coleta}

Test of Sensory Functions in Infants (TSFI) (DEGANGI; GREENSPAN, 2001) é um instrumento de triagem usado para identificar problemas de processamento sensorial em crianças e, quando usado em conjunto com outros instrumentos, a exemplo da Bayley Scale of Infant and Toddlers Development $-3^{R D}$ edition (Bayley III) (BAYLEY, 2006), traça o perfil do desenvolvimento funcional do bebê.

O TSFI fornece uma medida geral do processamento sensorial e da reatividade de lactentes de quatro aos 18 meses de vida, e contém cinco subdomínios: reação à pressão profunda; funçóes motoras adaptativas; integração visual-tátil; controle oculomotor, e reação a estímulos vestibulares. Cada um dos subdomínios apresenta uma pontuaçáo específica, que é somada ao final para apresentaçáo do resultado total. O resultado total possui três pontos de corte: processamento sensorial normal, em risco ou deficiente, de acordo com o intervalo de idades: quatro a seis meses, sete a nove meses, dez a 12 meses e 13 a 18 meses.

Bayley Scales of Infant and Toddler Development $-3^{R D}$ edition (Bayley III) (BAYLEY, 2006) é utilizada para avaliar o desenvolvimento de crianças de um a 42 meses de idade e é composta pelos subtestes: cognição, linguagem (comunicação receptiva e expressiva) e motricidade (fina e grossa). Para este estudo, foi utilizado unicamente o subteste cognitivo, que consta de 91 itens. A criança inicia a avaliação no item correspondente à sua faixa etária, mas se ela erra algum dos três primeiros itens, retorna ao item correspondente da idade anterior. A avaliação é encerrada após cinco erros consecutivos, como indica o manual.

Para a interpretação dos resultados, utilizou-se o escore composto, que apresenta média de 100 pontos e 
desvio padrão de 15 pontos, sendo o desenvolvimento cognitivo considerado adequado quando os resultados do escore composto variam entre 85 e 115 pontos.

\subsection{Procedimentos de avaliação}

Todos os lactentes foram avaliados no Ambulatório de Puericultura ou de Fonoaudiologia do HC-UFPE. Foi utilizado um formulário de pesquisa desenvolvido especificamente para este estudo, a fim de colher as variáveis biológicas e sociodemográficas. Os registros de prontuário, resumos de alta da maternidade e caderneta da criança foram a fonte de informaçáo para as condiçôes biológicas do lactente, enquanto que as condiçôes sociodemográficas foram registradas por meio de entrevista com as mães ou responsáveis.

Um estudo piloto foi realizado com dez lactentes para verificar a padronização das avaliaçóes e a qualidade dos formulários de pesquisa, para que alteraçôes pudessem ser feitas em tempo hábil, de forma a melhorar a qualidade da coleta dos dados. As examinadoras foram devidamente treinadas para a aplicação dos instrumentos, a fim de minimizar os erros de administração dos testes. O TSFI foi aplicado por duas terapeutas ocupacionais, com conhecimento da teoria de integração sensorial, e a Bayley III foi aplicada por uma fonoaudióloga e por uma terapeuta ocupacional, ambas especialistas em desenvolvimento infantil.

A confiabilidade da avaliação do desenvolvimento cognitivo e do processamento sensorial foi realizada entre dois observadores, em aproximadamente $10 \%$ da amostra. O coeficiente de correlação intraclasse (ICC) foi utilizado para avaliar a reprodutibilidade da escala Bayley III, por se tratar de uma variável contínua, em que o nível de concordância foi de 0,88 (IC 95\% 0,70-0,95; p < 0,001). Para o TSFI, por se tratar de uma variável categórica, foi utilizado o índice de Kappa, obtendo-se o resultado 0,40 para o escore total. A reprodutibilidade dos testes foi considerada excelente para a Bayley III e, entre regular e boa, para o TSFI.

\subsection{Análise dos dados}

Para garantir a consistência dos resultados, os dados foram registrados em formulários com questôes pré-codificadas e digitados através da dupla entrada no programa Epi info (versão 6.04), utilizando-se o subprograma validate, para minimizar os possíveis erros de digitação.

O índice de desenvolvimento cognitivo é uma variável contínua e, para fins de análise foi categorizado em "normal" e "atraso", de acordo com o resultado do escore composto. Os lactentes com escore composto $\geq 85$ foram considerados com desenvolvimento cognitivo normal e aqueles que obtiveram um escore $\leq 84$ foram classificados como apresentando atraso. Para possibilitar a análise da avaliaçáo do processamento sensorial (variável categórica), em que as crianças podem ser classificadas em: deficiente, em risco e normal, a categoria "deficiente", devido ao seu pequeno número, foi agrupada ao grupo "em risco".

O teste do Qui-quadrado com correção de Yates foi empregado para examinar a associação entre as variáveis dicotômicas, utilizando-se o teste exato de Fisher, quando indicado. Adotou-se o intervalo de confiança de $95 \%$ e nível de significância estatística de $5 \%(p \leq 0,05)$.

\section{Resultados}

A caracterização da amostra encontra-se na Tabela 1. Dos 182 lactentes estudados, 91 (50\%) era do sexo feminino e 54 (30\%), pré-termo. A média de idade cronológica para os nascidos a termo foi 9,7 meses ( $\mathrm{DP}=2)$ e a média da idade corrigida para os nascidos pré-termo foi 9,6 meses ( $\mathrm{DP}=2)$, no momento da avaliação. A renda familiar per capita e a escolaridade materna náo diferiram significantemente entre os grupos.

A Tabela 2 apresenta a associação entre a idade gestacional ao nascer com o processamento sensorial e com a cogniçã́o. Em ambos os grupos, 48 lactentes $(26,4 \%)$ apresentaram o TSFI total em risco e deficiente, e seis $(3,3 \%)$ tiveram atraso cognitivo. Verificou-se uma frequência significantemente maior de processamento sensorial em risco e deficiente entre os lactentes nascidos pré-termo (37\%), quando comparados aos nascidos a termo (21,9\%). O atraso do desenvolvimento cognitivo não esteve associado à prematuridade.

As Tabelas 3 e 4 apresentam resultados da amostra como um todo. A Tabela 3 mostra associaçóes entre as variáveis biológicas, socioeconômicas e demográficas familiares com o processamento sensorial dos lactentes (TSFI total). Verifica-se que não foram encontradas associaçôes estatisticamente significantes entre as variáveis. Apenas as crianças que usaram ventilação mecânica assistida e CPAP mostraram uma maior frequência de processamento sensorial em risco ou deficiente, sendo essas associaçóes limítrofes. Tal resultado pode ter ocorrido devido ao pequeno número de recém-nascidos que foram submetidos a intervençóes intensivas. O percentual de crianças com processamento sensorial em risco 
Tabela 1. Caracterização da amostra dos 182 lactentes.

\begin{tabular}{|c|c|c|c|c|}
\hline \multirow{2}{*}{$\begin{array}{l}\text { Variáveis biológicas, socioeconômicas } \\
\text { e demográficas }\end{array}$} & \multicolumn{2}{|c|}{ Pré-Termo } & \multicolumn{2}{|c|}{ A Termo } \\
\hline & Média & DP & Média & DP \\
\hline Peso ao nascer (g) & 1832,3 & 755,5 & 3209,6 & 509,3 \\
\hline Idade Gestacional (semanas) & 33 & 3 & 39 & 1 \\
\hline \multirow[t]{2}{*}{ Idade na Avaliação (meses) } & 9,5 & 1,8 & 9,7 & 2 \\
\hline & $\mathbf{N}$ & $(\%)$ & $\mathrm{n}$ & $(\%)$ \\
\hline \multicolumn{5}{|l|}{ Sexo } \\
\hline Masculino & 23 & $(42,6)$ & 68 & $(53,1)$ \\
\hline Feminino & 31 & $(57,4)$ & 60 & $(46,9)$ \\
\hline \multicolumn{5}{|l|}{ Renda familiar per capita (SM*) } \\
\hline$\leq$ meio SM & 40 & $(74,1)$ & 87 & $(68,0)$ \\
\hline$>$ meio SM & 14 & $(25,9)$ & 41 & $(32,0)$ \\
\hline \multicolumn{5}{|l|}{ Escolaridade materna } \\
\hline Fundamental & 11 & $(20,4)$ & 33 & $(26,2)$ \\
\hline Médio & 41 & $(75,9)$ & 82 & $(65,1)$ \\
\hline Superior & 2 & $(3,7)$ & 11 & $(8,7)$ \\
\hline
\end{tabular}

*SM = salário mínimo.

Tabela 2. Associação da idade gestacional com o processamento sensorial e o desenvolvimento cognitivo de lactentes nascidos pré-termo e a termo.

\begin{tabular}{|c|c|c|c|c|c|c|c|}
\hline \multirow{3}{*}{ Idade Gestacional } & \multirow{2}{*}{\multicolumn{2}{|c|}{ Total }} & \multicolumn{4}{|c|}{ Processamento Sensorial (TSFI Total) } & \multirow{3}{*}{$\mathbf{P}$} \\
\hline & & & \multicolumn{2}{|c|}{ Em risco/Deficiente } & \multicolumn{2}{|c|}{ Normal } & \\
\hline & $\mathbf{N}$ & $(\%)$ & $\mathbf{N}$ & $(\%)$ & $\mathrm{n}$ & $(\%)$ & \\
\hline Pré-termo & 54 & $(29,7)$ & 20 & $(37,0)$ & 34 & $(63,0)$ & 0,05 \\
\hline A termo & 128 & $(70,3)$ & 28 & $(21,9)$ & 100 & $(78,1)$ & \\
\hline \multirow[t]{4}{*}{ Total } & 182 & $(100)$ & 48 & $(26,4)$ & 134 & $(73,6)$ & \\
\hline & \multirow{2}{*}{\multicolumn{2}{|c|}{ Total }} & \multicolumn{4}{|c|}{ Cognição (Bayley III) } & \multirow{3}{*}{$\mathbf{P}$} \\
\hline & & & \multicolumn{2}{|c|}{ Atraso $(\leq 84)$} & \multicolumn{2}{|c|}{ Normal $(\geq 85)$} & \\
\hline & $\mathbf{N}$ & $(\%)$ & $\mathbf{N}$ & $(\%)$ & $\mathrm{n}$ & $(\%)$ & \\
\hline Pré-termo & 54 & $(29,7)$ & 2 & $(3,7)$ & 52 & 96,3 & $1,0^{*}$ \\
\hline A termo & 128 & $(70,3)$ & 4 & $(3,1)$ & 124 & 96,9 & \\
\hline Total & 182 & $(100)$ & 6 & $(3,3)$ & 167 & $(91,7)$ & \\
\hline
\end{tabular}

*Teste exato de Fisher.

ou deficiente tendeu a aumentar progressivamente com o aumento da idade, isto é, quanto mais velha a criança, maior a ocorrência de comportamento em risco ou deficiente no TSFI total.

A Tabela 4 apresenta associação entre a avaliação do processamento sensorial (TSFI total) e seus subtestes com a cognição dos lactentes.

Verifica-se que a ocorrência de atraso cognitivo foi significantemente maior nas crianças com processamento sensorial em risco e deficiente para o TSFI total, quando comparadas com as que tiveram o processamento sensorial normal. Em relação aos subtestes do TSFI, observa-se que os lactentes com atraso cognitivo apresentaram uma frequência significantemente maior de alteração do processamento sensorial nos subtestes de função motora adaptativa e de integração tátil-visual, quando comparados com os que tiveram processamento sensorial normal.

\section{Discussão}

A revisão sistemática apresentada por Mitchell et al. (2015) mostrou que, até o momento, apenas quatro artigos sobre o processamento sensorial na populaçáo de prematuros foram publicados. Estes estudos mostram que a prematuridade apresenta repercussões negativas em diferentes áreas do processamento, as quais, por sua vez, interferem no comportamento dessas crianças diante da informação sensorial, sendo a maioria delas hiper-reativas a estímulos, especialmente visuais, táteis e auditivos.

Devido à escassez de pesquisas que examinam a associação do processamento sensorial de lactentes com a prematuridade e o desenvolvimento cognitivo, a presente pesquisa foi realizada objetivando responder a duas perguntas: a primeira, sobre a influência da prematuridade no processamento sensorial, e a 
Tabela 3. Associação entre variáveis biológicas, condições socioeconômicas e demográficas familiares com o processamento sensorial dos lactentes.

\begin{tabular}{|c|c|c|c|c|c|c|c|}
\hline \multirow{3}{*}{ Variáveis } & \multirow{2}{*}{\multicolumn{2}{|c|}{ Total }} & \multicolumn{5}{|c|}{ Processamento Sensorial (TSFI Total) } \\
\hline & & & \multicolumn{2}{|c|}{$\begin{array}{l}\text { Em risco/ } \\
\text { Deficiente }\end{array}$} & \multicolumn{2}{|c|}{ Normal } & \multirow[t]{2}{*}{$\mathbf{P}$} \\
\hline & $\mathbf{N}$ & $(\%)$ & $\mathbf{N}$ & $(\%)$ & $\mathrm{n}$ & $(\%)$ & \\
\hline \multicolumn{8}{|l|}{ Peso ao nascer (g) } \\
\hline$\leq 1500$ & 24 & $(13,4)$ & 7 & $(29,2)$ & 17 & 70,8 & 0,97 \\
\hline$>1500$ & 155 & $(86,6)$ & 41 & $(26,5)$ & 114 & 73,5 & \\
\hline \multicolumn{8}{|l|}{ Sexo } \\
\hline Masculino & 91 & (50) & 26 & $(28,6)$ & 65 & $(71,4)$ & 0,61 \\
\hline Feminino & 91 & $(50)$ & 22 & $(24,2)$ & 69 & $(75,8)$ & \\
\hline \multicolumn{8}{|l|}{ Hipóxia } \\
\hline Sim & 17 & $(9,7)$ & 5 & $(29,4)$ & 12 & $(70,6)$ & $0,78^{*}$ \\
\hline Não & 158 & $(90,3)$ & 43 & $(27,2)$ & 115 & $(72,8)$ & \\
\hline \multicolumn{8}{|c|}{ Hemorragia Intracraniana } \\
\hline Sim & 8 & $(4,5)$ & 4 & $(50,0)$ & 4 & $(50,0)$ & $0,22 *$ \\
\hline Não & 169 & $(95,5)$ & 44 & $(26,0)$ & 125 & $(74,0)$ & \\
\hline \multicolumn{8}{|c|}{ Doença da Membrana Hialina } \\
\hline Sim & 7 & $(4,0)$ & 3 & $(42,8)$ & 4 & $(57,2)$ & $0,40^{*}$ \\
\hline Não & 167 & $(96,0)$ & 45 & $(27,0)$ & 122 & $(73,0)$ & \\
\hline \multicolumn{8}{|c|}{ Ventilação Mecânica Assistida } \\
\hline Sim & 14 & $(8,0)$ & 7 & $(50,0)$ & 7 & $(50,0)$ & $0,06 *$ \\
\hline Não & 161 & $(92,0)$ & 41 & $(25,5)$ & 120 & $(74,5)$ & \\
\hline \multicolumn{8}{|l|}{ CPAP } \\
\hline Sim & 35 & $(20,0)$ & 14 & $(40,0)$ & 21 & $(60,0)$ & $0,10^{*}$ \\
\hline Não & 140 & $(80,0)$ & 34 & $(24,3)$ & 106 & $(75,7)$ & \\
\hline \multicolumn{8}{|l|}{ Convulsão } \\
\hline Sim & 4 & $(2,3)$ & 2 & $(50,0)$ & 2 & $(50,0)$ & $0,30^{*}$ \\
\hline Não & 172 & $(97,7)$ & 46 & $(26,7)$ & 126 & $(73,3)$ & \\
\hline \multicolumn{8}{|l|}{ Uso de Sonda } \\
\hline Sim & 40 & $(23,5)$ & 14 & $(35,0)$ & 26 & $(65,0)$ & 0,28 \\
\hline Não & 130 & $(76,5)$ & 32 & $(24,6)$ & 98 & $(75,4)$ & \\
\hline \multicolumn{8}{|c|}{ Idade na avaliação (meses) } \\
\hline 8 & 81 & $(44,5)$ & 16 & $(19,8)$ & 65 & $(80,2)$ & \\
\hline $9-11$ & 62 & $(34,1)$ & 18 & $(29,0)$ & 44 & $(71,0)$ & $0,05 * * *$ \\
\hline $12-15$ & 39 & $(21,4)$ & 14 & $(35,9)$ & 25 & $(64,1)$ & \\
\hline \multicolumn{8}{|c|}{ Renda per capita (SM**) } \\
\hline$\leq$ meio SM & 127 & $(69,8)$ & 32 & $(25,0)$ & 95 & $(75,0)$ & 0,71 \\
\hline$>$ meio SM & 55 & $(30,2)$ & 16 & $(29,0)$ & 39 & $(71,0)$ & \\
\hline \multicolumn{8}{|l|}{ Escolaridade materna } \\
\hline Fundamental & 45 & $(25,0)$ & 11 & $(22,9)$ & 34 & $(25,8)$ & 0,57 \\
\hline Médio & 122 & $(67,8)$ & 35 & $(72,9)$ & 87 & $(65,9)$ & \\
\hline Superior & 13 & $(7,2)$ & 2 & $(4,2)$ & 11 & $(8,3)$ & \\
\hline \multicolumn{8}{|l|}{ Mamou } \\
\hline Não & 6 & $(3,3)$ & 3 & $(50,0)$ & 3 & $(50,0)$ & $0,19^{*}$ \\
\hline Sim & 175 & $(96,7)$ & 45 & $(25,7)$ & 130 & $(74,3)$ & \\
\hline
\end{tabular}

*Teste exato de Fisher; **SM = Salário Mínimo; ***Qui-quadrado de tendência.

segunda, se existe associação entre o processamento sensorial e o desenvolvimento cognitivo de lactentes.

Quanto à primeira pergunta, os resultados do estudo sugerem que a prematuridade é um fator em risco para as alteraçôes do processamento sensorial, mostrando que os lactentes nascidos prematuros tiveram maior frequência de comportamentos considerados em risco e deficiente, de acordo com o TSFI. A literatura refere que condiçóes de risco biológico, como a prematuridade e os procedimentos invasivos realizados na UTI neonatal, necessários para a manutenção da estabilidade clínica do lactente, 
Tabela 4. Associação entre o processamento sensorial (TSFI total) e seus subtestes com o desenvolvimento cognitivo de lactentes.

\begin{tabular}{|c|c|c|c|c|c|c|c|}
\hline \multirow{3}{*}{$\begin{array}{c}\text { Processamento Sensorial } \\
\text { (TSFI Total) }\end{array}$} & \multicolumn{6}{|c|}{ Cognição (Bayley III) } & \multirow{3}{*}{$\mathbf{P}$} \\
\hline & \multicolumn{2}{|c|}{ Total } & \multicolumn{2}{|c|}{ Atraso $(\leq 84)$} & \multicolumn{2}{|c|}{ Normal $(\geq 85)$} & \\
\hline & $\mathbf{N}$ & $(\%)$ & $\mathrm{n}$ & $(\%)$ & $\mathrm{n}$ & $(\%)$ & \\
\hline Em risco e deficiente & 48 & $(26,4)$ & 4 & $(8,3)$ & 44 & $(91,7)$ & $0,04 *$ \\
\hline Normal & 134 & $(73,6)$ & 2 & $(1,5)$ & 132 & $(98,5)$ & \\
\hline \multicolumn{8}{|l|}{ Subtestes do TSFI } \\
\hline \multicolumn{8}{|l|}{ Reação à pressão profunda } \\
\hline Em risco e deficiente & 47 & $(25,8)$ & 2 & $(4,3)$ & 45 & $(95,7)$ & $0,65^{*}$ \\
\hline Normal & 135 & $(74,2)$ & 4 & $(3,0)$ & 131 & (97) & \\
\hline \multicolumn{8}{|l|}{ Função motora adaptativa } \\
\hline Em risco e deficiente & 49 & $(26,9)$ & 4 & $(8,2)$ & 45 & $(91,8)$ & $0,04 *$ \\
\hline Normal & 133 & $(73,1)$ & 2 & $(1,5)$ & 131 & $(98,5)$ & \\
\hline \multicolumn{8}{|l|}{ Integração visual-tátil } \\
\hline Em risco e deficiente & 54 & $(29,7)$ & 5 & $(9,3)$ & 49 & $(90,7)$ & $0,009 *$ \\
\hline Normal & 128 & $(70,3)$ & 1 & $(0,8)$ & 127 & $(99,2)$ & \\
\hline \multicolumn{8}{|l|}{ Controle oculomotor } \\
\hline Em risco e deficiente & 5 & $(2,7)$ & 0 & $(0,0)$ & 5 & $(100)$ & $1,0^{*}$ \\
\hline Normal & 177 & $(97,3)$ & 6 & $(3,4)$ & 171 & $(96,6)$ & \\
\hline \multicolumn{8}{|c|}{ Reação à estimulação vestibular } \\
\hline Em risco e deficiente & 28 & $(15,4)$ & 2 & $(7,1)$ & 26 & $(92,9)$ & $0,23 *$ \\
\hline Normal & 154 & $(84,6)$ & 4 & $(2,6)$ & 150 & $(97,4)$ & \\
\hline
\end{tabular}

*Teste exato de Fisher.

podem aumentar a predisposição dos mesmos a alteraçóes no neurodesenvolvimento (WIENER et al., 1996; CASE-SMITH; BUTCHER; REED, 1998; ALS et al., 2004; AYRES, 2005; ZOMIGNANI; ZAMBELLI; ANTONIO, 2009; BART et al., 2011; WICKREMASINGHE et al., 2013).

Als et al. (2004) afirmam que os cuidados prestados na UTI neonatal influenciam as funçóes dos sistemas motor e de autorregulaçâo, além de outros aspectos associados ao neurocomportamento, como o estado de alerta e o limiar de resposta aos estímulos sensoriais do ambiente. Esse achado pode justificar uma maior frequência de alteraçóes do processamento sensorial nos lactentes nascidos prematuros.

Em relação à segunda pergunta do estudo, sobre a associação entre o processamento sensorial e o desenvolvimento cognitivo, os resultados mostraram uma associação estatisticamente significante entre o processamento sensorial de lactentes com o resultado da escala cognitiva da Bayley III. Lactentes com escore composto cognitivo $\leq 84$, que é considerado atraso, apresentaram uma frequência significantemente maior entre aqueles com processamento sensorial em risco ou deficiente. Esse resultado difere do encontrado por Case-Smith, Butcher e Reed (1998), que observaram independência entre a responsividade sensorial, utilizando a Sensory Rating Scale (SRS) e os índices de desenvolvimento medidos através da escala Bayley II. Esse achado pode estar relacionado ao uso de instrumentos diferentes, tanto para avaliar o processamento sensorial como para avaliar o desenvolvimento cognitivo.

Da amostra de lactentes pré-termo, apenas dois (3,7\%) apresentaram atraso cognitivo, de acordo com a escala de Bayley III. Esse resultado pode ser devido ao fato de que, ao se corrigir a idade dos prematuros, muitos atingem os níveis adequados de desenvolvimento, compensando algumas dificuldades. Além disso, a maioria deles (65\%) teve idade gestacional de menor risco (>32 semanas - dado não apresentado), o que pode também explicar uma menor frequência de complicaçóes e morbidades neonatais e, consequentemente, fraca associação entre estas e os índices da Bayley III.

Pelo desenho do estudo, não é possível se estabelecer uma relação causal, ou seja, determinar se as alteraçôes de processamento sensorial precoce acarretariam alteraçóes cognitivas posteriormente, sendo esta uma limitaçáo metodológica relevante. Por isso, estudos prospectivos são necessários para se conhecer tanto o valor preditivo do TSFI como para acompanhar os efeitos em médio e longo prazo das alteraçóes de processamento sensorial na vida de crianças nascidas prematuras. Além disso, existe uma tendência das alteraçôes de processamento sensorial se tornarem mais frequentes com o avançar da idade (autores). 
A influência da prematuridade no desenvolvimento do processamento sensorial infantil também foi estudada por Wiener et al. (1996). Eles verificaram maior frequência de processamento sensorial em risco e deficiente, de acordo com o TSFI, em lactentes nascidos pré-termo, quando comparados aos a termo, entre sete e 18 meses de vida. Todos os lactentes tinham o desenvolvimento mental e motor normal de acordo com a escala de Bayley II, porém não foi realizada análise estatística para testar a associação entre os índices da Bayley e o resultado do TSFI.

Analisando-se os subtestes do TSFI separadamente e sua associação com o atraso cognitivo, observou-se diferença estatisticamente significante para a função motora adaptativa e a integraçáo visual-tátil, que apresentam componentes do desenvolvimento cognitivo precoce (BEE, 2003). Esses subtestes envolvem diretamente a aquisição de ações mentais e físicas que passam pelo processo de aprendizado; logo, quando as crianças apresentaram atraso cognitivo na escala Bayley III, esses subtestes do TSFI mostraram-se mais frequentemente alterados. Os demais itens do TSFI que verificam como a criança percebe a intensidade e a natureza do estímulo sensorial náo mostraram relação com a cognição.

Utilizando-se o TSFI como teste de triagem, observa-se que apenas seu escore total é insuficiente para verificar a associação de fatores de risco e as funçôes do processamento sensorial. Uma análise detalhada dos seus subtestes permite estabelecer com mais cautela associaçóes, sendo possível verificar funçôes específicas do processamento sensorial, como modulação e praxia. Outra consideração relevante sobre o teste é o caráter interpretativo que o mesmo possui, uma vez que a interpretaçáo da reação da criança frente ao estímulo sensorial apresentado irá depender da experiência e do conhecimento do examinador sobre o processamento sensorial. Uma hipótese levantada com a análise dos subtestes é que nem todas as funçôes apresentadas no TSFI têm relação com a cogniçáo, como relatado por Case-Smith, Butcher e Reed (1998), mostrando a necessidade de novos estudos de acompanhamento que confirmem ou não tal relaçáo.

$\mathrm{O}$ presente estudo mostrou que quanto maior a idade do lactente no momento da avaliação, maior a frequência de TSFI em risco e deficiente, sendo essa tendência significante. Achados semelhantes foram encontrados nos estudos de Bart et al. (2011) e de Wickremasinghe et al. (2013). A relação entre o processamento sensorial com a idade da criança pode ser explicada pelo seguinte fato: quanto mais velha for a criança, mais tarefas ela deve realizar, levando à percepção de alguns comportamentos associados a alteraçóes do processamento sensorial mais tardiamente.

Outras pesquisas realizadas com crianças mais velhas mostraram a influência da alteração do processamento sensorial nas atividades ocupacionais, como alimentação, controle esfincteriano e sono e vigília (FARROW; COULTHARD, 2012; POLLOCK; METZ; BARABASH, 2014; VASAK et al., 2015). $\mathrm{Na}$ idade escolar, as crianças com problemas de processamento e integração da informação sensorial tendem a desenvolver dificuldades na escrita, na manutenção da atenção para o aprendizado acadêmico e na tendência ao isolamento, por evitar esportes de grupo (AYRES, 1972; DEMAIO-FELDMAN, 1994; BLANCHE, 2005). Bakker e Moulding (2012), Farrow e Coulthard (2012) e Nakagawa et al. (2016) mostram, em seus estudos, que alteração no processamento sensorial pode ainda estar associado a sintomas psicológicos negativos e dificuldades de temperamento em crianças e adultos.

Blanche (2005), ao avaliar uma população de adultos com sinais de processamento sensorial deficiente na infância, observou que essas alteraçóes se mantêm ao longo da vida e influenciam as escolhas ocupacionais dos indivíduos, que podem ter suas atividades limitadas, se não tratado.

\section{Conclusão}

Os lactentes nascidos prematuros apresentaram mais frequentemente sinais sugestivos de alteração do processamento sensorial e aqueles que apresentaram alteração do processamento sensorial tiveram maior chance de ter atraso do desenvolvimento cognitivo. A prematuridade, isoladamente, entretanto, não parece ter a mesma influência no desenvolvimento cognitivo. Esse resultado sugere um alerta para a inclusão de uma triagem para o processamento sensorial no programa de acompanhamento de lactentes nascidos prematuros e mostra como Terapeutas Ocupacionais, com certificação e experiência na avaliação do processamento sensorial, podem fazer parte dessa equipe.

\section{Referências}

ALS, H. et al. Early experience alters brain function and structure. Pediatrics, Springfield, v. 113, n. 4, p. 846857, 2004.

AYRES, A. J. Improving academic scores trough sensory integration. Journal of Learning Desabilities, London, v. 5, n. 6, p. 338-343, 1972.

AYRES, A. J. Sensory integration and praxis tests. Los Angeles: Western Psychological Services, 1989. 
AYRES, A. J. Sensory integration and the child: understanding hidden sensory challenges. Los Angeles: Western Psychological Services, 2005.

BAKKER, K.; MOULDING, R. Sensory processing sensitivity, dispositional mindfulness and negative psychological symptoms. Personality and Individual Differences, London, v. 53, n. 3, p. 341-346, 2012.

BART, O. et al. Prediction of participation and sensory modulation of late preterm infants at 12 months: A prospective study. Research in Developmental Disabilities, New York, v. 32, n. 6, p. 2732-2738, 2011.

BAYLEY, N. Bayley scales of infant and toddler development: administration manual. New York: PSYCHORP, 2006.

BEE, H. Desenvolvimento cognitivo I: estrutura e processo. In: BEE, H. A criança em desenvolvimento. Porto Alegre: Artmed, 2003. p. 191-227.

BLANCHE, E. I. Déficit de integración sensorial: efectos a largo plazo sobre la ocupación y el juego. Revista Chilena de Terapia Ocupacional, Santiago, n. 5, p. 1-6, 2005.

BRASIL. Ministério da Saúde. Secretaria de Atenção à Saúde. Departamento de Açóes Programáticas Estratégicas. Atenção humanizada ao recém-nascido de baixo peso: método Canguru. Brasília: Editora do Ministério da Saúde, 2011.

BUNDY, A. C. et al. How does sensory processing dysfunction affect play? American Journal of Occupational Therapy, Boston, v. 61, n. 2, p. 201-208, 2007.

CASE-SMITH, J.; BUTCHER, L.; REED, D. Parents' report of sensory responsiveness and temperament in preterm infants. American Journal of Occupational Therapy, Boston, v. 52, n. 7, p. 547-555, 1998.

DEGANGI, G. Pediatric disorders of regulation in affect and behavior: a therapist's guide to assessment and treatment. California: Elsevier, 2000.

DEGANGI, G.; GREENSPAN, S. I. Test of Sensory Function in Infants (TSFI). Los Angeles: Western Psychological Services, 2001.

DEMAIO-FELDMAN, D. Somatosensory processing abilities of very low-birth weight infants at school age. American Journal of Occupational Therapy, Boston, v. 48, n. 7, p. 639-645, 1994.

ENGEL-YEGER, B. Sensory processing patterns and daily activity preferences of Israeli children. Canadian Journal of Occupational Therapy, Toronto, v. 75, n. 4, p. 220-229, 2008.
FARROW, C. V.; COULTHARD, H. Relationships between sensory sensitivity, anxiety and selective eating in children. Appetite, London, v. 58, n. 3, p. 842-846, 2012.

KOENIG, K. P.; RUDNEY, S. G. Performance challenges for children and adolescents with difficulty processing and integrating sensory information: a systematic review. American Journal of Occupational Therapy, Boston, v. 64, n. 3, p. 430-442, 2010.

MITCHELL, A. W. et al. Sensory processing disorder in children ages birth -3 years born prematurely: a systematic review. American Journal of Occupational Therapy, Boston, v. 69, n. 1, p. 1-11, 2015.

NAKAGAWA, A. et al. Relations between temperament, sensory processing, and motor coordination in 3-year-old children. Frontiers is Psychology, Pully, v. 7, n. 623, p. 1-7, 2016.

PEDROSA, C.; CAÇOLA, P.; CARVALHAL, M. I. M. M. Fatores preditores do perfil sensorial de lactentes dos 4 aos 18 meses de idade. Revista Paulista de Pediatria, São Paulo, v. 33, n. 2, p. 160-166, 2015.

POLLOCK, M. R.; METZ, A. E.; BARABASH, T. Association between dysfunctional elimination syndrome and sensory processing disorder. American Journal of $O c$ cupational Therapy, Boston, v. 68, n. 4, p. 472-477, 2014.

REEVES, G. D.; CERMAK, S. A. Disorders of praxis. In: BUNDY, A. C.; LANE, S. J.; MURRAY, E. A. Sensory integration: theory and practice. Philadelphia: F. A. Davis, 2002. p. 71-100.

VASAK, M. et al. Sensory processing and sleep in typically developing infants and toddlers. American Journal of Occupational Therapy, Boston, v. 69, n. 4, p. 1-8, 2015.

WHITE, B. P. et al. An examination of the relationships between motor and process skills and scores on the sensory profile. American Journal of Occupational Therapy, Boston, v. 61, n. 2, p. 154-160, 2007.

WICKREMASINGHE, A. C. et al. Children born prematurely have atypical sensory profiles. Journal of Perinatology, New York, v. 33, n. 8, p. 631-638, 2013.

WIENER, A. S. et al. Sensory processing of infants born prematurely or with regulatory disorders. Physical and Occupationl Therapy in Pediatrics, New York, v. 16, n. 4, p. 1-18, 1996.

ZOMIGNANI, A. P.; ZAMBELLI, H. J. L.; ANTONIO, M. A. R. G. M. Desenvolvimento cerebral em recém-nascidos prematuros. Revista Paulista de Pediatria, São Paulo, v. 27, n. 2, p. 198-203, 2009.

\section{Contribuição dos Autores}

Flávia Regina trabalhou na concepção da pesquisa, coleta e processamento dos dados, interpretação dos resultados e redação do texto. Sophie Helena realizou a orientação de todo o processo de pesquisa e de elaboração e revisão do texto. Marilia participou da análise e interpretação dos dados e revisão final do texto. Todos os autores aprovaram a versão final do texto. 